Die „Hamburger Rechtsstudien“ erscheinen in zwangloser Folge und sind einzeln in jeder Buchhandlung käuflich

Bisher sind erschienen:

Heft 1: Der Begriff des Versicherungsfalles in der Seeversicherung. Von Dr. $F$. Alexander Bene. Groß-Oktav. 75 Seiten. 1928.

RM 4.05

Heft 2: Die Bedeutung des Interesses für die Verïußerung der versicherten Sache. Von Dr. Hermann Heinrich Elkan. Groß-Oktav. 58 Seiten. 1928. RM 3.60

Heft 3: Aktiensonderdepot und Legitimationsübertragung. Von Dr. Günther Frohner. Groß-Oktav, 121 Seiten. 1929. RM 6.30

Heft 4: Die Gewinnversicherung. Von Dr. Helmut Winkler. Groß-Oktav. 31 Seiten. 1930.

RM 1.80

Heft 5: Der Konnossement-Teilschein. Von Dr. Heinz Behlert. GroB-Oktay, 79 Seiten. $1930 . \quad$ RM 4.50

Heft 6: Die Order-Police. Von Dr. Alexander N. Tsirintanis. GroB-Oktav. 95 Seiten. $1930 . \quad$ RM 5.40

Heft 7: Reine Konnossemente gegen Revers. Von Dr. Robert Lion. Groß-Oktav. 78 Seiten. 1930.

RM. 4.50

Heft 8: Versicherung für Rechnung wen es angeht. Von Dr. Helmuth Embden. GroB-Oktav. 39 Seiten, 1930.

RM 2.70

Heft 9: Die guten Sitten in der arbeitsrechtlichen Rechtsprechung nach dem Kriege. Von Dr. Fritz Oettinger. Groß-Oktav. 84 Seiten. 1931 RM 4.5̃0

Heft 10: Wandlung und Minderung bei einer Mehrheit von Käufern oder Verkïu. fern. Von Dr. Hans Wogatzky. GroB-Oktav. 115 Seiten. 1931. RM 6.-

Heft 11: Das Versicherungs-Zertifikat. Von Dr. Rudolf Nothmann. GroB-Oktav. 96 Seiten. 1932. RM 5.

Heft 12: Die Versicherung der Havariegrosse-Sehäden. Von Dr. Hans Cramer. Groß-Oktav. 56 Seiten. 1932. RM 3.-

Heft 13: Die Staatshaftung für den Hamburger Hafenlotsen. Von Dr. Erwin Mumssen. Groß-Oktav. 110 Seiten. 1932. RM 5.-

Heft 14: Gleichberechtigung der Geschlechter im künftigen Elternrecht. Von Dr. Charlotte Cohn. Groß-Oktav. XI u. 56 Seiten, 1932. RM 3.50

Heft 15: Die Speditionsversicherung in den Allgemeinen Deutschen Spediteurbedingungen. Von Dr. Willi Schiering. Groß-Oktav. 74 Seiten. 1932.

RM 4.-

Heft 16: Quellenkritische Studien zur Bessergebotsklausel (in diem addictio) im römischen Kaufrecht. Von Dr. jur. Harald Sieg. Groß-Oktav. 43 Seiten. 1933.

RM 3.-

Heft 17: Kostfrachtgeschäft uud laufende Versicherung. Von Dr. Detlev Himer. Groß-Oktav. 46 Seiten. 1933.

RM 3.-

Heft 18: ,Acatholicus“, eine Untersuchung über die Stellung der getauften und exkommunierten Christen im geltenden kanonischen Recht. Von Dr. Walter Bohm. Groß-Oktav. 59 Seiten. 1933.

RM 3.-

Heft 19: Beitrüge zur Lehre von den subjektiven Anrechtselementen im Strafrecht. Von Dr. Rudolf Sieverts. Groß-Oktav, ca. 240 Seiten. 1934. RM 10.-

Heft 20: Die vorvertragliche Anzeigepflicht des Versicherungsnehmers. Von Dr. Klaus Koops. Groß-Oktav. 52 Seiten. 1934.

RM 3.-

Heft 21: Das Zustandekommen des Versicherungsvertrages, eine rechtsvergleichende Darstellung. Von Dr. Heinz Hagemann. 
Die vorvertragliche Anzeigepflicht des Versicherungsnehmers.

Eine rechtsvergleichende Darstellung.

$$
\text { von }
$$

\section{Dr. KLAUS KOOPS}

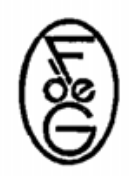

Hamburg

Friederichsen, de Gruyter \& Co. m. b. H.

$\begin{array}{llll}1 & 9 & 3 & 4\end{array}$ 
Christiansdruck, Hamburg 36 